\title{
INCREMENTO EN EL RENDIMIENTO Y SUS COMPONENTES BAJO RIEGO NORMAL Y RESTRINGIDO DE VARIEDADES MEXICANAS DE TRIGO
}

\section{GAINS IN YIELD AND ITS COMPONENTS UNDER NORMAL AND LIMITED IRRIGATION OF MEXICAN WHEAT CULTIVARS}

\author{
Sara L. Paquini-Rodríguez ${ }^{1}$, Ignacio Benítez-Riquelme ${ }^{1}$, Héctor E. Villaseñor- \\ Mir $^{2 \star}$, Abel Muñoz-Orozco ${ }^{1}$ y Humberto Vaquera-Huerta ${ }^{3}$
}

\begin{abstract}
1Programa de Genética del Posgrado de Recursos Genéticos y Productividad y ${ }^{3}$ Programa de Socioeconomía, Estadística e Informática, Colegio de Postgraduados. 56230, Montecillo, Texcoco, Estado de México. ${ }^{2}$ Programa de Trigo, Campo Experimental Valle de México, Instituto Nacional de Investigaciones Forestales, Agrícolas y Pecuarias. Km 13.5 Carr. Los Reyes-Lechería. 56250, Coatlinchán, Texcoco, Estado de México, México.
\end{abstract}

*Autor para correspondencia (villaseñor.hector@inifap.gob.mx)

\section{RESUMEN}

El agua que se utiliza para el riego en las zonas trigueras de México tiene limitantes, y dado el incremento en el consumo de trigo (Triticum aestivum L.), la presión sobre el recurso hídrico aumentará. El objetivo de la presente investigación fue evaluar bajo riego normal y restringido, el desempeño en rendimiento de grano y sus componentes de 16 variedades de trigo harinero liberadas desde la década de 1940 hasta el 2015. Durante el ciclo de cultivo otoño-invierno (2014 a 2015) se establecieron cuatro experimentos, dos en Montecillo, Edo. de México y dos en Celaya, Guanajuato, en una fecha de siembra óptima y otra tardía. El diseño experimental fue bloques al azar con arreglo de parcelas divididas donde la parcela grande fue el nivel de riego, y la pequeña las variedades. El riego restringido no fue lo suficientemente severo como para detectar diferencias significativas en el rendimiento y sus componentes, excepto para la fecha tardía en Celaya. Los análisis combinados y para fechas de siembra revelaron diferencias significativas entre los niveles de riego para todas las variables en estudio, de las cuales las más afectadas por restringir el riego fueron rendimiento de grano, biomasa aérea, y número de granos por $\mathrm{m}^{2}$, con una reducción de $20.3,16.8$, y 14.5 $\%$, respectivamente. La ganancia del rendimiento de grano fue $1.21 \%$ anual $\left(41.77 \mathrm{~kg} \mathrm{ha}^{-1}\right)$, que representó un aumento acumulado de $2.8 \mathrm{tha}^{-1}$ en 67 años. Bajo riego normal el progreso de $1.10 \%$ fue menor que bajo riego restringido $(1.35 \%)$, lo que indica que en el futuro el rendimiento podría mantenerse con menos consumo de agua y esto permitiría ampliar el área sembrada usando el mismo volumen de agua.

Palabras clave: Rendimiento de grano, riego restringido, Triticum aestivum, variedades mexicanas de trigo.

\section{SUMMARY}

Availability of irrigation water for wheat-producing areas in México is limited, but wheat (Triticum aestivum L.) demand keeps increasing, and thus water needs are greater. This study tested 16 bread wheat cultivars released from 1940 to 2015 and recorded their grain yield and its components. Four field trials were established during the 2014 - 2015 Fall - Winter crop season. Two trials were sowed in Montecillo, state of México and two in Celaya, Guanajuato, and two sowing dates were tested: optimum and late. The experiments were arranged in random blocks under a split plot design. Main plots were irrigation levels, and small plots were cultivars. Under limited irrigation, most treatment combinations did not show significant differences in yield and its components, except for late-sown wheat in Celaya. Combined and sowing dates analyses revealed significant differences among irrigation levels for all the variables recorded. Limited irrigation affected grain yield, aerial biomass, and number of grains per $\mathrm{m}^{2}$; their reduction was $20.3,16.8$, and
$14.5 \%$, respectively. Annual average yield increased $1.21 \%\left(41.77 \mathrm{~kg} \mathrm{ha}^{-1}\right)$; in 67 years, the accumulated yield increment is $2.8 \mathrm{t} \mathrm{ha}^{-1}$. Rate of yield improvement decreased under normal irrigation $(1.10 \%)$ compared to the rate under limited irrigation (1.35\%). These results suggest that less water may produce the same yield in the future, and current water volume could support a wider cultivated area.

Index words: Grain yield, limited irrigation, Triticum aestivum, Mexican bread wheat cultivars.

\section{INTRODUCCIÓN}

En México el déficit de agua se localiza principalmente en el noroeste, norte y centro del país, donde se ubican los estados productores de trigo (Triticum aestivum L.): Sonora, Baja California, Chihuahua y Guanajuato, cuya precipitación acumulada anual oscila entre 56 y $200 \mathrm{~mm}$ en los dos primeros estados, y 292 y $548 \mathrm{~mm}$ respectivamente para el tercero y cuarto (CONAGUA, 2013; SMN, 2015). En las principales zonas trigueras $58 \%$, aproximadamente, de los acuíferos presentan déficit de agua (CONAGUA, 2015) mientras que las presas ahí ubicadas varían de 5.5 a 73.6 $\%$ de su capacidad (CONAGUA, 2013), por lo que todas las regiones hidrológico-administrativas correspondientes a esas zonas están clasificadas con un alto grado de presión sobre el recurso hídrico. Por otra parte, habrá que sumar al déficit hídrico el incremento de la temperatura, ya que el Grupo Intergubernamental de Expertos sobre el Cambio Climático pronostican que en México la temperatura aumentará de 0.5 a $1.5^{\circ} \mathrm{C}$ en el periodo 2016 a 2035 y la precipitación disminuirá de 0 a 20 \% (IPCC, 2013).

Desde los años setenta se vislumbraba un agravamiento de la disponibilidad del agua para sembrar trigo, por lo que se iniciaron los trabajos de mejoramiento genético encaminados a lograr la resistencia a diferentes niveles limitantes de humedad edáfica (Villaseñor et al., 2009). Desde entonces, el desarrollo de las nuevas variedades se ha basado en la aplicación de métodos combinados de mejoramiento 
como la selección masal y el genealógico. El Centro Internacional de Mejoramiento de Maíz y Trigo (CIMMYT) y el Instituto Nacional de Investigaciones Forestales Agrícolas y Pecuarias (INIFAP) generaron líneas para áreas de riego o temporal (secano) a partir de trigos importados y criollos, de cruzas de trigos de invierno con trigos de primavera, y de cruzas de trigos mejorados con especies silvestres; todos evaluados en diferentes altitudes y latitudes bajo riego reducido o en áreas de temporal (Huerta-Espino et al., 2011).

En la selección se han considerado rasgos relacionados con la resistencia a sequía como el peso de mil granos (Lopes et al., 2012b) y se han incorporado criterios fisiológicos (Reynolds et al., 2012), como en la línea elite Sokoll que mostró mayores valores de índice de cosecha, biomasa y eficiencia en el uso de agua que sus progenitores, y éstos se cruzaron estratégicamente (Lopes y Reynolds, 2011). A pesar de la complejidad del mejoramiento genético, hay evidencias de ganancias en el rendimiento de grano tanto para condiciones de riego limitado (Lopes et al., 2012a) como para condiciones óptimas de crecimiento (Nalley et al., 2010). Ante el desabasto de $55.2 \%$ en el grano de trigo panificable (CANIMOLT, 2014), es urgente aumentar el rendimiento con un uso más eficiente del agua de riego o en ambientes de temporal de baja precipitación.

En este contexto, resulta valioso explorar el germoplasma elite del pasado y del presente para visualizar el comportamiento, a través del tiempo, de características cuya heredabilidad y correlación es alta con el rendimiento de grano bajo riego normal, y para saber cómo esta asociación es afectada por el riego restringido. Por ello, el objetivo de la presente investigación fue evaluar el comportamiento genético de variedades de trigo liberadas desde la década de 1940 hasta el 2015, bajo condiciones de riego normal y de riego restringido.

\section{MATERIALES Y MÉTODOS}

Durante el ciclo de cultivo otoño-invierno de 2014 a 2015 se establecieron cuatro experimentos, dos en Montecillo, Edo. de México y dos en Celaya, Guanajuato, en una fecha de siembra óptima y otra tardía. El diseño experimental fue de bloques al azar con arreglo en parcelas divididas con dos repeticiones donde la parcela grande fue el nivel de riego, y la pequeña las variedades. Los niveles de riego fueron riego normal y restringido. La unidad experimental consistió de cuatro surcos de $2 \mathrm{~m}$ de longitud separados a $0.3 \mathrm{~m}$, y la parcela útil fue el total de la unidad experimental (2.4 $\mathrm{m}^{2}$ ). La densidad de siembra para los cuatro experimentos fue de $100 \mathrm{~kg} \mathrm{ha}^{-1}$.

En los experimentos de Celaya el riego restringido con- sistió en dejar de aplicar el último riego (etapas 65 a 70 de Zadoks, según la variedad, Zadoks et al., 1974) de cuatro riegos en la primera fecha de siembra, y suspender los dos últimos (etapas 45 a 50 de Zadoks) en la segunda fecha de siembra. En Montecillo la restricción del riego se llevó a cabo a partir de la etapa de elongación del tallo (etapas 35 a 40 de Zadoks). Las variedades, facilitadas por el INIFAP, fueron dos por cada década desde 1940 al 2020 (Cuadro 1), y del total se seleccionaron las variedades más representativas de su respectiva década.

La fertilización se aplicó al voleo al momento de la siembra para los experimentos en Montecillo, a una dosis de $80 \mathrm{~N}-40 \mathrm{P}-00 \mathrm{~K}$ con urea y superfosfato de calcio triple. Para los experimentos en Celaya, la dosis fue 120N-60P-00K aplicada al momento de la siembra, y antes del segundo riego se aplicó la dosis 120N-00P-00K. Las malezas en las dos localidades fueron controladas con la aplicación

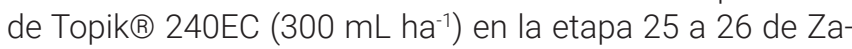
doks, Esteron® $47 \mathrm{M}\left(750 \mathrm{~mL}^{\circ} \mathrm{ha}^{-1}\right)$ en la etapa 29 a 30 de Zadoks. En Montecillo, en la primera fecha de siembra se usó Karate Zeon® ${ }^{\circledR}\left(700 \mathrm{~mL}\right.$ ha $\left.^{-1}\right)$ en la etapa 34 de Zadoks contra Shizaphis graminum con incidencia de $10 \%$. En las dos localidades la prevención y control de roya lineal (Puccinia striiformis Westend) se realizó con la aplicación de Folicur ${ }^{\circledR}\left(600 \mathrm{~mL} \mathrm{ha}^{-1}\right)$ en las etapas 57 a 59 y 73 a 75 de Zadoks.

Se determinaron las propiedades físicas y químicas del suelo en cada localidad, así como las constantes de humedad punto de marchitez permanente y capacidad de campo mediante los métodos de la membrana de presión y de la olla respectivamente. Se tomaron muestras de suelo cada $7 \mathrm{~d}$ en Montecillo a una profundidad de 0 a $10 \mathrm{~cm}$ y de 10 a $30 \mathrm{~cm}$, y cada $10 \mathrm{~d}$ en Celaya a una profundidad de 0 a $30 \mathrm{~cm}$, con el fin de medir la humedad actual con el método gravimétrico y la fórmula: \% de humedad $=(($ Peso suelo húmedo - Peso suelo seco) / Peso suelo seco) x 100.

Se registró la temperatura máxima y mínima del aire en periodos de $10 \mathrm{~d}$, y la precipitación pluvial acumulada en el mismo lapso desde la siembra hasta la cosecha. Las variables medidas fueron: 1) Peso de mil granos (PMG), obtenido con el promedio de tres muestras de 200 granos tomados al azar y multiplicado por 5; 2) Biomasa aérea, calculada con la ecuación $\mathrm{BIO}=(\mathrm{RG}+25 \mathrm{RE} / \mathrm{PU}) / \mathrm{IC}$, donde $R G$ es el rendimiento de grano de la parcela, $R E$ es el peso de grano de 25 espigas, $\mathrm{PU}$ es la parcela útil en $\mathrm{m}^{2}$ e IC el índice de cosecha calculado mediante la expresión $\mathrm{IC}=\mathrm{RE} / \mathrm{RB}$, donde RB se determinó en una muestra de 25 tallos cortados al ras del suelo a lo largo de los dos surcos centrales de cada unidad experimental, los tallos fueron puestos a secar en un invernadero durante $4 \mathrm{~d}$ y luego se pesaron; 3) Rendimiento de grano de los cuatro 
surcos transformado a $\mathrm{kg} \mathrm{ha}^{-1}$; 4) Número de espigas por $\mathrm{m}^{2}$ calculado con la ecuación EMPC $\left.=\mathrm{BIO} / \mathrm{RB} ; 5\right)$ Número de granos por $\mathrm{m}^{2}$ calculado con la ecuación $\mathrm{GPMC}=(\mathrm{RG} /$ PMG) x 100; y 6) Tasa de llenado de grano calculada como $T L L G=R G /($ días a madurez-días a espigamiento).

Se hicieron análisis de varianza para cada experimento, para fechas de siembra y combinado, con el procedimiento GLM del programa SAS (Statistical Analysis System para Windows versión 9.0) donde las repeticiones se tomaron como un factor aleatorio en los dos últimos análisis. Para las variables con significancia estadística se realizaron pruebas de comparación de medias con la prueba de Tukey al 0.05 de probabilidad. Con el programa Minitab 17 se llevó a cabo un análisis de regresión simple entre la década de liberación de las variedades y el rendimiento obtenido bajo riego normal y restringido, y entre la década de liberación de las variedades y el rendimiento resultado de sumar el rendimiento bajo riego normal y el rendimiento bajo riego restringido.

Para obtener la tasa o porcentaje de avance genético, el valor del coeficiente de regresión se dividió entre el valor de la ordenada al origen, y el resultado se multiplicó por cien. Además, se utilizó el método de regresión simple entre la década de liberación y cada una de las variables.

\section{RESULTADOS Y DISCUSIÓN}

\section{Condiciones ambientales de los experimentos}

Los suelos de las dos localidades fueron arcillosos, aunque el de Celaya con una mayor proporción de arcilla (70.4 $\%$ vs. $53.5 \%$ en Montecillo), lo que le permitió retener más humedad, y en consecuencia disminuir el número de riegos durante el ciclo de cultivo (Figuras 1 y 2).

En la condición de riego restringido en la primera fecha de siembra en Celaya, la humedad edáfica disminuyó al punto de marchitez permanente (PMP) a los 103 d después de la siembra (dds) (en el periodo de llenado de grano) a pesar de la presencia de lluvias, y se mantuvo así hasta la cosecha (Figura 1). Es precisamente en esta región que durante el periodo de llenado de grano se omite el último riego, lo que genera una sequía terminal.

Actualmente, los mejoradores en El Bajío desarrollan variedades a las cuales se les pueda limitar a dos o tres los riegos, aunque con dos riegos el rendimiento puede abatirse en $13 \%$ comparado con el rendimiento obtenido con tres riegos (Solís-Moya et al., 2013). En esta localidad y para la segunda fecha de siembra, el PMP ocurrió alrededor de los 80 dds justo en floración y llenado de grano de la mayoría de las variedades, y así prosiguió hasta la cosecha (Figura 1).

\begin{tabular}{|c|c|c|c|}
\hline Genotipo & Década & Año de liberación & Condición \\
\hline Kentana 48 & \multirow{2}{*}{$1941-1950$} & 1948 & Riego \\
\hline Chapingo 48 & & 1948 & Riego \\
\hline Yaktana 54C & \multirow{2}{*}{$1951-1960$} & 1954 & Riego \\
\hline Lerma Rojo & & 1955 & Riego \\
\hline Lerma Rojo S64 & \multirow{2}{*}{$1961-1970$} & 1964 & Riego \\
\hline Siete Cerros T66 & & 1966 & Riego \\
\hline Salamanca S75 & \multirow{2}{*}{$1971-1980$} & 1975 & Riego \\
\hline Pavón F76 & & 1976 & Riego \\
\hline Seri M82 & \multirow{2}{*}{ 1981-1990 } & 1982 & Riego \\
\hline Temporalera M87 & & 1987 & Temporal \\
\hline Baviácora M92 & \multirow{2}{*}{ 1991-2000 } & 1992 & Riego \\
\hline Cortázar S94 & & 1994 & Riego \\
\hline Roelfs F2007 & \multirow{2}{*}{$2001-2010$} & 2007 & Riego \\
\hline Nana F2007 & & 2007 & Temporal \\
\hline Borlaug 100 F2014 & \multirow{2}{*}{$2011-2020$} & 2014 & Riego \\
\hline Bacorehuis F2015 & & 2015 & Riego \\
\hline
\end{tabular}




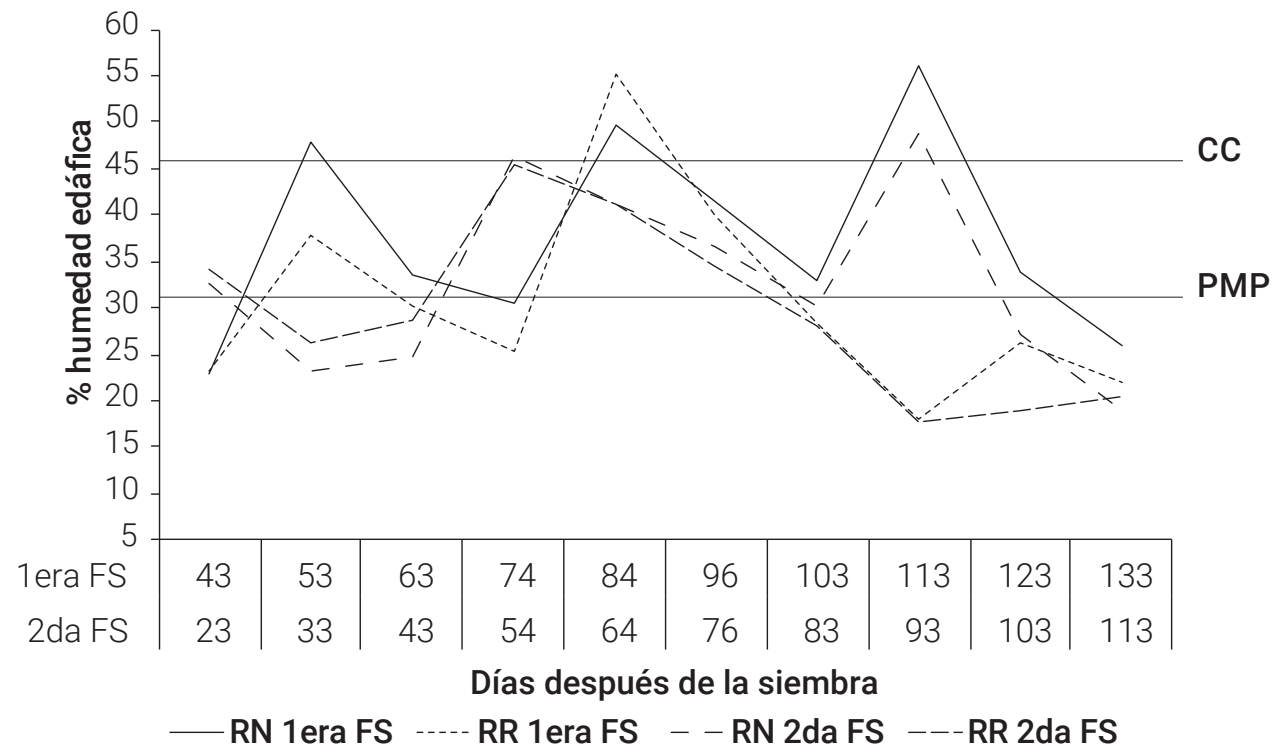

Figura 1. Contenido de humedad edáfica a una profundidad de 0 a $30 \mathrm{~cm}$ bajo riego normal (RN) y restringido (RR) de la primera (1a FS) y segunda fecha de siembra (2a FS) en Celaya, Guanajuato. CC = capacidad de campo; PMP = punto de marchitez permanente.

Para la primera y segunda fecha de siembra en Montecillo, la lluvia atípica que se presentó después de haber suspendido el riego ( 407.7 mm de precipitación desde la primera semana de marzo hasta la primera semana de junio), y la escasa retención de agua del suelo provocaron altibajos de humedad edáfica. Aunque la humedad del suelo no estuvo disponible a partir de los 33 y 43 dds bajo riego restringido y hasta antes de la cosecha (Figura 2) para la primera y segunda fecha de siembra respectivamente, la lluvia atenuó el efecto de la limitación de riego, por lo que la sequía se consideró moderada.

\section{Análisis de varianza de cada experimento}

La sequía inducida no fue lo suficientemente severa para poder detectar diferencias significativas entre niveles de riego en la mayoría de las variables. En la segunda fecha de siembra en Celaya se detectó un efecto significativo del nivel de riego en el rendimiento porque, por un lado, el estrés por sequía comenzó fenológicamente antes que en la primera fecha. Esto es, entre más tiempo dure la sequía y entre menos agua disponible en el suelo, el rendimiento es menor (Akram, 2011; Farooq et al., 2014; Orta et al., 2002; Zhang et al., 2016).

Al considerar que los efectos combinados de sequía y temperatura pueden ser aditivos (Kaur y Behl, 2010) o más que aditivos (Prasad et al., 2011), se podría inferir que la interacción de la sequía terminal y las temperaturas máximas y mínimas más altas incidentes en el periodo de llenado de grano, cuando se limitó el riego (alrededor de $2.5^{\circ} \mathrm{C}$ más alta que en la primera fecha), fue determinante para detec- tar la diferencia en las variables debida al nivel de riego; esto no se reveló en Montecillo porque el cambio en temperaturas en el mismo periodo de llenado de grano fue de apenas $0.8^{\circ} \mathrm{C}$ en promedio, entre la primera y segunda fecha de siembra.

El factor "variedad" mostró un efecto diferente para todas las variables en todos los experimentos, mientras que la interacción variedad por nivel de riego fue significativa para peso de mil granos (PMG), granos por metro cuadrado (GPMC) y rendimiento de grano (RG) en la primera fecha de siembra en Montecillo y en la segunda fecha de siembra en Celaya. Dicha interacción también fue significativa para PMG, GPMC y tasa de llenado de grano (TLLG) en la segunda fecha de siembra en Montecillo.

\section{Análisis de varianza para fechas de siembra en cada localidad}

Este análisis detectó cambios significativos en todas las variables para ambas localidades debido al nivel de riego. También mediante el análisis de varianza, Shamsi y Kobraee (2011) detectaron efectos significativos del estrés por sequía en el rendimiento y todos sus componentes. La fecha de siembra afectó el comportamiento de las variedades en Celaya y por consiguiente todas las características, pero en Montecillo la fecha de siembra no produjo cambios en granos por $\mathrm{m}^{2}$ (GPMC) ni en rendimiento de grano (RG) posiblemente porque en Montecillo la diferencia de temperaturas máximas y mínimas entre las fechas de siembra durante el periodo de llenado de grano no fue mayor de $0.8^{\circ} \mathrm{C}$ en promedio. 
En lugares de climas semicálidos (como Celaya) y cálidos donde el aumento de temperaturas coincide con el llenado del grano, el retraso de la fecha de siembra disminuye el rendimiento y sus componentes porque se acortan las fases del desarrollo del trigo, que a su vez impactan el desarrollo del grano (Suleiman et al., 2014). De acuerdo con la comparación de medias con la prueba de Tukey $(a=0.05)$, en Celaya la fecha de siembra tardía causó reducción de rendimiento de grano $(R G)$, peso de mil granos (PMG), biomasa aérea (BIO), espigas por $\mathrm{m}^{2}$ (EPMC), granos por $\mathrm{m}^{2}$ (GPMC) y tasa de llenado de grano (TLLG) en 34.9, 18.9, $31.5,9.0,20.2$, y $14.2 \%$, respectivamente.

\section{Promedios del rendimiento}

En el Cuadro 2 se comparan los rendimientos obtenidos en cada nivel de riego por cada fecha de siembra y localidad, y cada fecha de siembra por localidad. Como ya se destacó, hubo diferencias significativas entre riego normal y restringido en la segunda fecha de siembra en Celaya y entre fechas de siembra en Celaya.

\section{Análisis de varianza combinado de los cuatro experimentos}

Los factores riego, variedad, ambiente (combinación de localidad y fecha de siembra), y la interacción variedad por ambiente, produjeron efectos significativos $(P \leq 0.05)$ en todas las variables (Cuadro 3). Según la comparación de medias con la prueba de Tukey $(a=0.05)$, al pasar de riego normal a riego restringido las variedades de trigo disminu- yeron su rendimiento de grano, peso de mil granos, biomasa aérea, espigas por $\mathrm{m}^{2}$ granos por $\mathrm{m}^{2}$, y tasa de llenado de grano en $20.3,6.5,16.8,11.5,14.5$, y $9.1 \%$, respectivamente.

Estos resultados concuerdan con los de Shamsi y Kobraee (2011), quienes concluyeron que el estrés por sequía redujo significativamente el rendimiento, el peso de mil granos y el número de granos por $\mathrm{m}^{2}$. Según Ivanova y Tsenov (2011), la reducción del rendimiento en 30 \% se debió a disminuciones en peso de granos por espiga (cerca de $40 \%$ ), número de granos por espiga (cerca de $29 \%$ ) y tamaño de los granos (cerca de $15 \%$ ) provocada por el déficit hídrico. Madani et al. (2010) explicaron que el efecto de la sequía se manifiesta a través de una menor tasa de llenado de grano y reparto de materia seca, porque se restringe la demanda de fotoasimilados.

\section{Tendencia de la ganancia del rendimiento}

La ganancia anual del rendimiento de grano promedio estimada de los cuatro experimentos fue de $1.21 \%$ (41.77 $\left.\mathrm{kg} \mathrm{ha}^{-1}\right)(P<0.001)$ (Figura 3), que en 67 años de trabajo continuo y a partir de las variedades liberadas en el año de 1948, ello representó un aumento acumulado de $2.8 \mathrm{t} \mathrm{ha}^{-1}$ (81\% de ganancia total). El coeficiente de determinación reveló que la década de liberación de las variedades contribuyó significativamente a explicar el rendimiento de grano. Este resultado es semejante al progreso estimado por Nalley et al. (2010) de 1.53 t totales (38.25 kg ha-1 por año) con el uso de variedades liberadas de 1962 a 2002 que suman 40 años, y con base en los datos del programa de

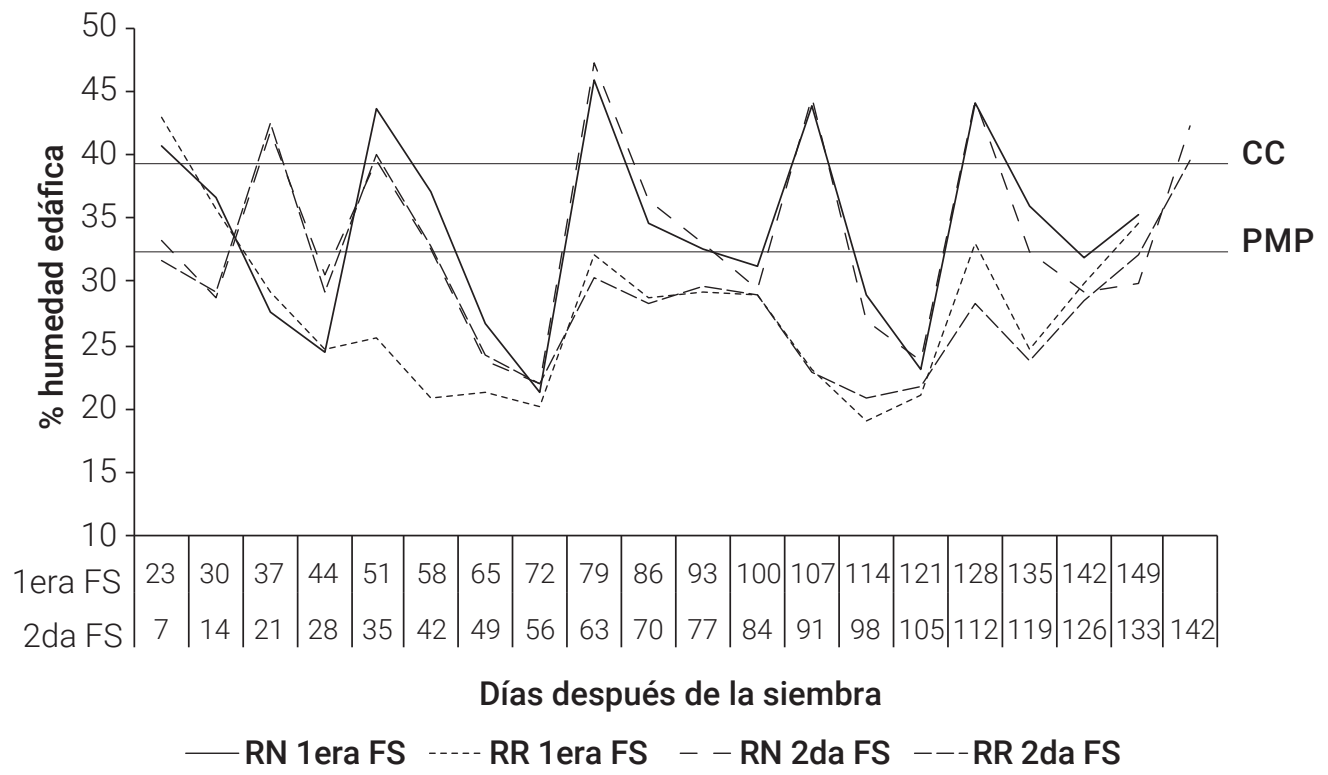

Figura 2. Contenido de humedad edáfica bajo riego normal (RN) y restringido (RR) de la primera (1a FS) y segunda fecha de siembra (2a FS) en Montecillo, Edo. de México de 0 a $10 \mathrm{~cm}$ de profundidad. $\mathrm{CC}=$ capacidad de campo; $\mathrm{PMP}=$ punto de marchitez permanente. 
mejoramiento del CIMMYT instalado en el Valle del Yaqui.

Recientemente Lopes et al. (2012a) reportaron una tasa de ganancia de rendimiento menor ( $0.7 \%$ anual, $35 \mathrm{~kg}$ ha1) que la de la presente investigación, pero considerando el periodo de 1977 a 2008 (30 años); la regresión que los investigadores muestran coincide con la de la Figura 3, lo que indica que las variedades más modernas rinden más que las antiguas, y lo mismo sucede en condiciones estresantes por calor y sequía. Es importante resaltar que en los cuatro experimentos de la presente investigación se mantuvo un control total de las royas (incidencia máxima de $10 \%$ en la variedad más susceptible Baviácora M92), situación que de no haberse hecho los avances genéticos habrían sido mayores, como le sucedió a Sayre et al. (1998) al evaluar el rendimiento de grano con control y sin control de la roya, ya que de acuerdo con Singh et al. (2005) una de las aportaciones relevantes del mejoramiento genético de trigo en México ha sido la resistencia genética a estos patógenos.

La tasa anual de progreso bajo riego restringido (1.35\%) mayor que la obtenida bajo riego normal (1.10\%) (Figura 4), indica que en el futuro el rendimiento bajo riego restringido podría ser cercano al rendimiento bajo riego normal. Por el contrario, Lopes et al. (2012a) reportaron que las tasas

Cuadro 2. Rendimiento medio de grano $\left(\mathrm{kg} \mathrm{ha}^{-1}\right)$ de variedades de trigo en dos niveles de riego y en dos fechas de siembra, en dos localidades (Celaya, Guanajuato y Montecillo, Edo. de México).

\begin{tabular}{|c|c|c|c|c|c|c|}
\hline FS /Localidad & $\mathrm{RN}$ & $\mathrm{RR}$ & DIF & DMS & $P$ & \% Reducción \\
\hline & \multicolumn{6}{|c|}{ Celaya } \\
\hline Primera fecha & 5542.3 & 4856.5 & 685.8 & 756.79 & 0.0599 & 12.4 \\
\hline Segunda fecha & 3717.7 & 3051.2 & 666.5 & 347.01 & 0.0143 & 17.9 \\
\hline DIF & 1824.6 & 1805.3 & & & & \\
\hline DMS $(0.05)^{\dagger}$ & 196.5 & 266.2 & & & & \\
\hline$P$ & 0.0001 & 0.0001 & & & & \\
\hline \multirow[t]{2}{*}{ \% Reducción } & 32.9 & 37.2 & & & & \\
\hline & \multicolumn{6}{|c|}{ Montecillo } \\
\hline Primera fecha & 7303.6 & 5480.9 & 1822.7 & 1970.8 & 0.0577 & 24.9 \\
\hline Segunda fecha & 7180.0 & 5531.3 & 1648.7 & 1850.8 & 0.0618 & 22.9 \\
\hline DIF & 123.6 & 50.4 & & & & \\
\hline DMS $(0.05)^{\dagger}$ & 338.43 & 366.2 & & & & \\
\hline$P$ & 0.4620 & 0.7810 & & & & \\
\hline \% Reducción & 1.7 & 0.91 & & & & \\
\hline
\end{tabular}

Cuadro 3. Análisis de varianza combinado de cuatro experimentos de trigo establecidos en dos sitios, Celaya, Guanajuato y Montecillo, Edo. de México, durante el ciclo otoño-invierno 2014-2015.

\begin{tabular}{|c|c|c|c|c|c|c|c|}
\hline \multirow{2}{*}{ Variable } & \multicolumn{6}{|c|}{ Cuadrados medios } & \multirow[b]{2}{*}{ CV (\%) } \\
\hline & V & NR & $\mathrm{AMB}$ & $V \times A M B$ & $V \times N R$ & Error & \\
\hline$P M G$ & $381.74 *$ & $357.45^{\star}$ & $1173.89 *$ & $14.27 \star$ & 4.32 & 4.25 & 5.89 \\
\hline $\mathrm{BIO}$ & $6.39 \times 10^{6 *}$ & $4.15 \times 10^{7 \star}$ & $5.14 \times 10^{7 *}$ & $8.67 \times 10^{5 \star}$ & $3.62 \times 10^{5}$ & $4.07 \times 10^{5}$ & 14.55 \\
\hline EPMC & $621.31 *$ & $3535.04^{\star}$ & $4004.74^{\star}$ & $202.43^{\star}$ & 80.02 & 92.39 & 15.85 \\
\hline GPMC & $8.79 \times 10^{7 \star}$ & $3.48 \times 10^{8 \star}$ & $4.12 \times 10^{8 \star}$ & $1.36 \times 10^{7 \star}$ & $7.12 \times 10^{6 \star}$ & $4.00 \times 10^{6}$ & 13.37 \\
\hline TLLG & $8642.50 *$ & $8229.66^{\star}$ & $22,859.51^{\star}$ & $1271.53^{\star}$ & 304.68 & 232.63 & 12.77 \\
\hline$R G$ & $2.12 \times 10^{7 *}$ & $9.30 \times 10^{7 \star}$ & $1.28 \times 10^{8 *}$ & $2.36 \times 10^{6 *}$ & $9.30 \times 10^{5}$ & $5.97 \times 10^{5}$ & 14.49 \\
\hline
\end{tabular}

$\mathrm{AMB}=$ ambiente o experimento; $\mathrm{NR}=$ nivel de riego; $\mathrm{V}=$ variedad; $\mathrm{V} \times \mathrm{AMB}=$ interacción variedad por ambiente; $\mathrm{V} \times \mathrm{NR}=$ interacción variedad por nivel de riego; $\mathrm{PMG}=$ peso de mil granos; $\mathrm{BIO}=$ biomasa en $\mathrm{g} \mathrm{m}^{-2} ; \mathrm{EPMC}=$ espigas por $\mathrm{m}^{2} ; \mathrm{GPMC}=$ granos por $\mathrm{m}^{2} ; \mathrm{TLLG}=$ tasa de llenado de grano; $\mathrm{RG}=$ rendimiento de grano en $\mathrm{kg}$ ha $^{-1} ;{ }^{*}$ significancia a $\mathrm{P}=0.05 ; \mathrm{CV}=$ coeficiente de variación. 


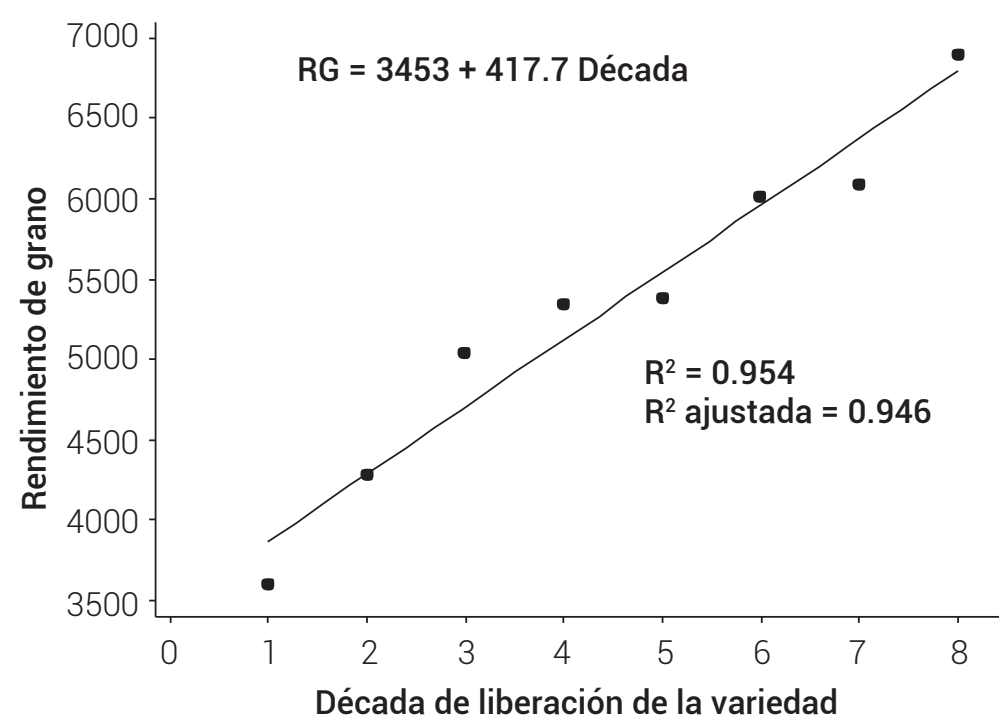

Figura 3. Regresión lineal simple del rendimiento de grano (RG) sobre las décadas de liberación de las variedades, en promedio de los cuatro experimentos. Celaya, Guanajuato y Montecillo, Edo. de México, ciclo otoño-invierno de 2014 - 2015.

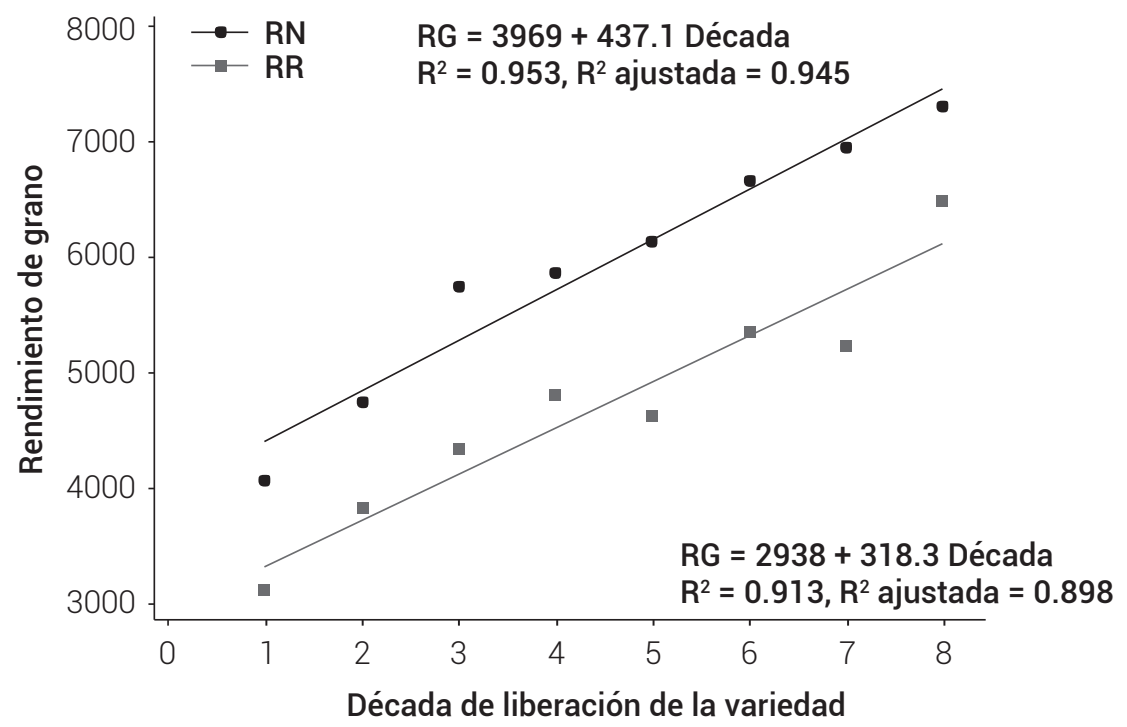

Figura 4. Relación entre la década de liberación de las variedades y el rendimiento de grano (RG) bajo riego normal (RN) y restringido (RR) como promedio de los cuatro experimentos. Celaya, Guanajuato y Montecillo, Edo. de México, ciclo otoñoinvierno de 2014 - 2015.

de ganancia son menores en ambientes relacionados con el estrés por sequía (alrededor de $0.7 \%$ por año), y con la combinación de sequía y calor (alrededor de $0.5 \%$ por año), que en ambientes con suficiente humedad edáfica o altamente rendidores (alrededor de $0.9 \%$ por año).

Los contrastantes resultados de ambas investigaciones posiblemente se deban a que se consideraron genotipos diferentes (excepto Seri M82), unos prove- nientes del CIMMYT y otros del INIFAP, y porque el periodo evaluado de mejoramiento fue más amplio en este estudio. La presente investigación pone de manifiesto que el trabajo de mejoramiento en México ha sido eficaz para aumentar continuamente el rendimiento de trigo en condiciones limitantes y óptimas de humedad edáfica. Blum (2011) ratificó el hecho de que con el mejoramiento genético se han logrado ganancias en el rendimiento del trigo y otros cultivos bajo condiciones de estrés por 
sequía a nivel mundial.

\section{Desempeño agronómico del trigo a través de las décadas en función del nivel del riego}

La regresión de biomasa aérea y la regresión de peso de mil granos, cada una contra tiempo, son cuadráticas y cóncavas tanto en riego normal como en riego restringido (Figuras 6 y 8), lo que indica que las variedades, década tras década, son cada vez mejores en estos rasgos y sobre todo en la última década, es decir, tienen un crecimiento exponencial. La regresión de peso de mil granos que reportan Lopes et al. (2012a) es lineal porque dicha característica heredada ha sido mejorada a través del tiempo en México, además está asociada con el rendimiento de grano.

Para variedades españolas e italianas, Álvaro et al. (2008) reportaron que el peso seco del cultivo (biomasa aérea) ha disminuido por efecto del mejoramiento desde antes de 1945 al 2000, porque también se ha reducido la altura de planta. La relación que guarda la tasa de llenado de grano (TLLG) y granos por $\mathrm{m}^{2}$ (GPMC) cada una con la década de liberación, tanto en riego normal como restringido, es lineal y positiva al igual que rendimiento de grano (Figuras 4 y 7 ). La mejora de la tasa de llenado de grano fue indirecta, es decir, se debió a mayor peso de grano, y no porque el periodo de llenado de grano de las variedades modernas sea más corto que el de las variedades antiguas.

En el caso del estudio mencionado de Álvaro et al. (2008), el mejoramiento no indujo cambio a lo largo de los años en la tasa de llenado de grano porque tampoco lo indujo en el peso de grano ni en la duración del llenado de grano. Por su parte, Isidro et al. (2011) también observaron un mayor número de granos por $\mathrm{m}^{2}$ con el paso de los años y lo relacionaron con una mejor partición de asimilados y un periodo más largo de llenado de grano. Todavía hay una brecha de los valores de los componentes del rendimiento entre riego normal y restringido, pero de acuerdo con las regresiones (Figuras 4 a 8) que pueden ayudar a predecir el comportamiento futuro de las variables, la tasa de llenado de grano y el número de granos por $\mathrm{m}^{2}$ tenderán a tener valores semejantes bajo riego normal y riego restringido, porque sus pendientes en riego restringido son mayores que en riego normal. Por su comportamiento a través de las décadas, se podría inferir que el peso de mil granos, la biomasa aérea y el número de granos por $\mathrm{m}^{2}$ han contribuido al rendimiento.

La característica número de espigas por $\mathrm{m}^{2}$ (EPMC) tuvo una relación cuadrática negativa con la década de liberación bajo riego normal (Figura 9), lo cual se traduce como una disminución continua con el paso de los años y sugiere que esta característica no ha contribuido al rendimiento de grano. Bajo riego restringido la regresión de EPMC no pudo ajustarse y por ello no se presenta ni la gráfica ni la ecuación. En contraste, de acuerdo con Sánchez-García et al. (2013), el número de espigas por $\mathrm{m}^{2}$ fue uno de los dos componentes del rendimiento de trigo harinero más afectados por el mejoramiento genético en España durante el

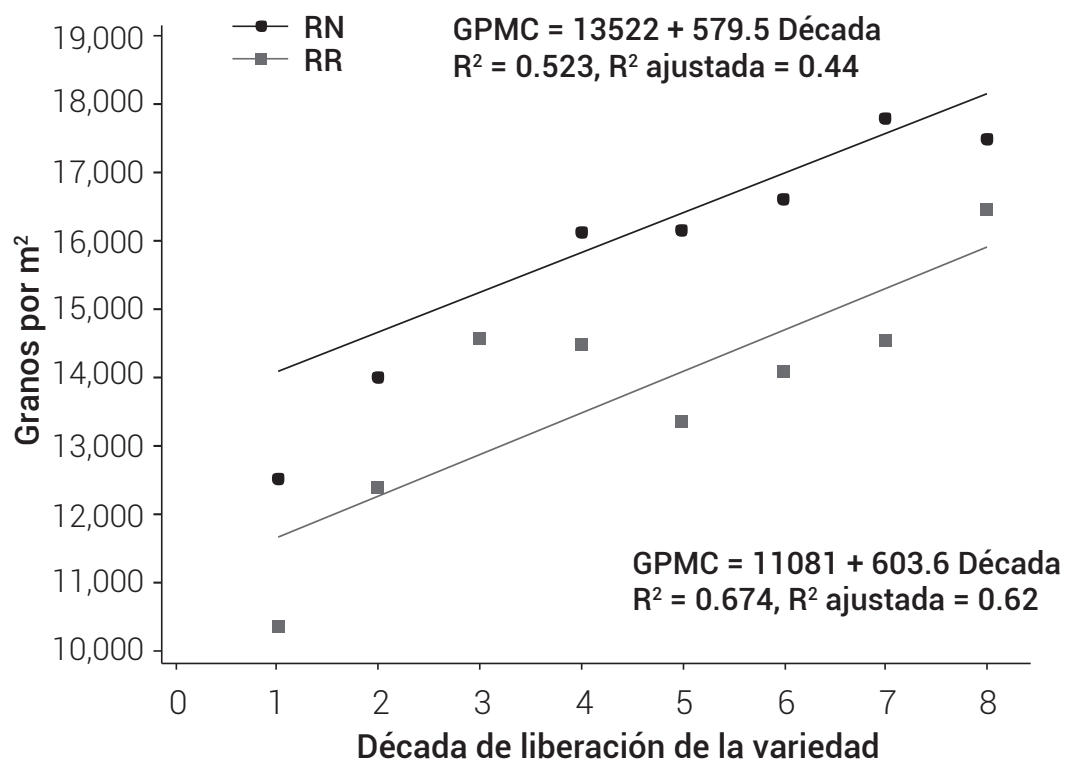

Figura 5. Relación entre la década de liberación de las variedades y granos por $\mathrm{m}^{2}$ (GPMC) bajo riego normal (RN) y restringido (RR) como promedio de los cuatro experimentos. Celaya, Guanajuato y Montecillo, Edo. de México, ciclo otoñoinvierno de 2014 - 2015. 
siglo XXI, ya que tuvo incremento de $30 \%$ por año. Sin embargo, dichos investigadores manifestaron que hubo cambios significativos del número de espigas por $\mathrm{m}^{2}$ solamente en uno de los cuatro años de evaluación, lo cual lo relacionan "con una variación grande del componente genotipo por am- biente de la varianza registrada para número de espigas por $\mathrm{m}^{2 \prime}$.

Los mejoradores tienen oportunidad de seguir aumentando el rendimiento al disminuir la brecha que hay entre riego

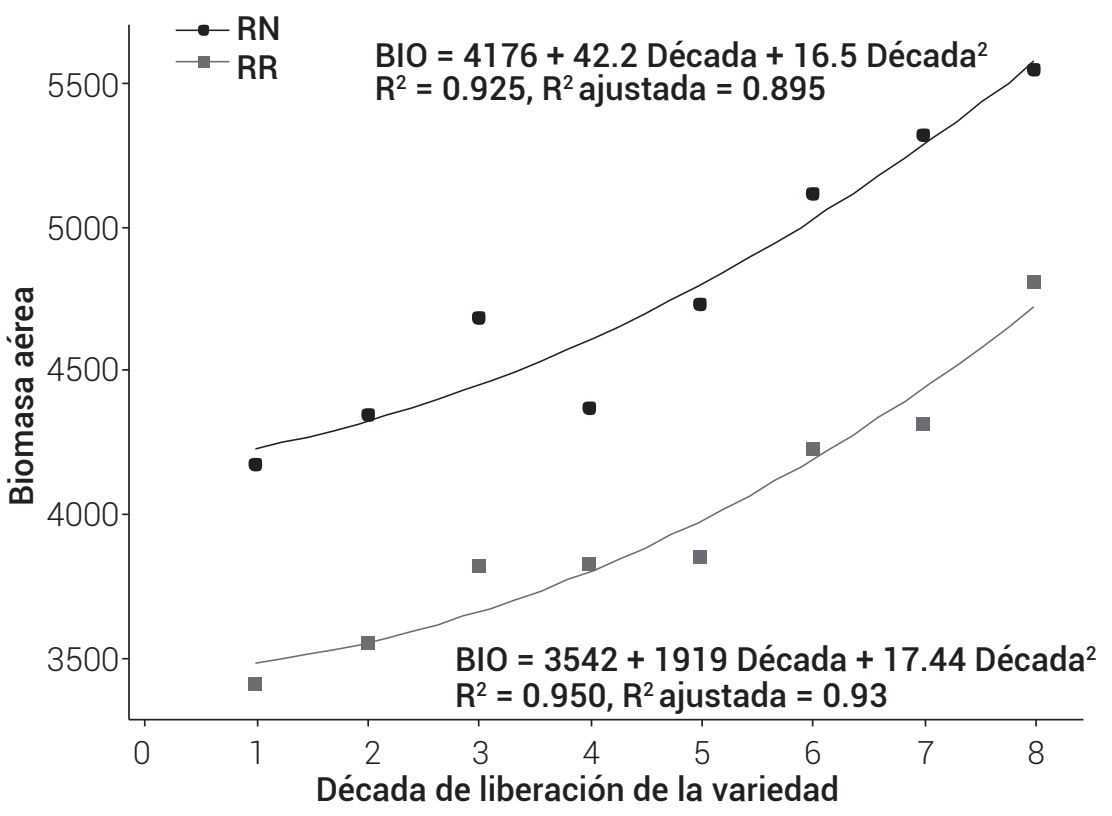

Figura 6. Relación entre la década de liberación de las variedades y biomasa aérea (BIO en $\mathrm{g} \mathrm{m}^{-2}$ ) bajo riego normal (RN) y restringido (RR) como promedio de los cuatro experimentos. Celaya, Guanajuato y Montecillo, Edo. de México, ciclo otoño-invierno de 2014 - 2015.

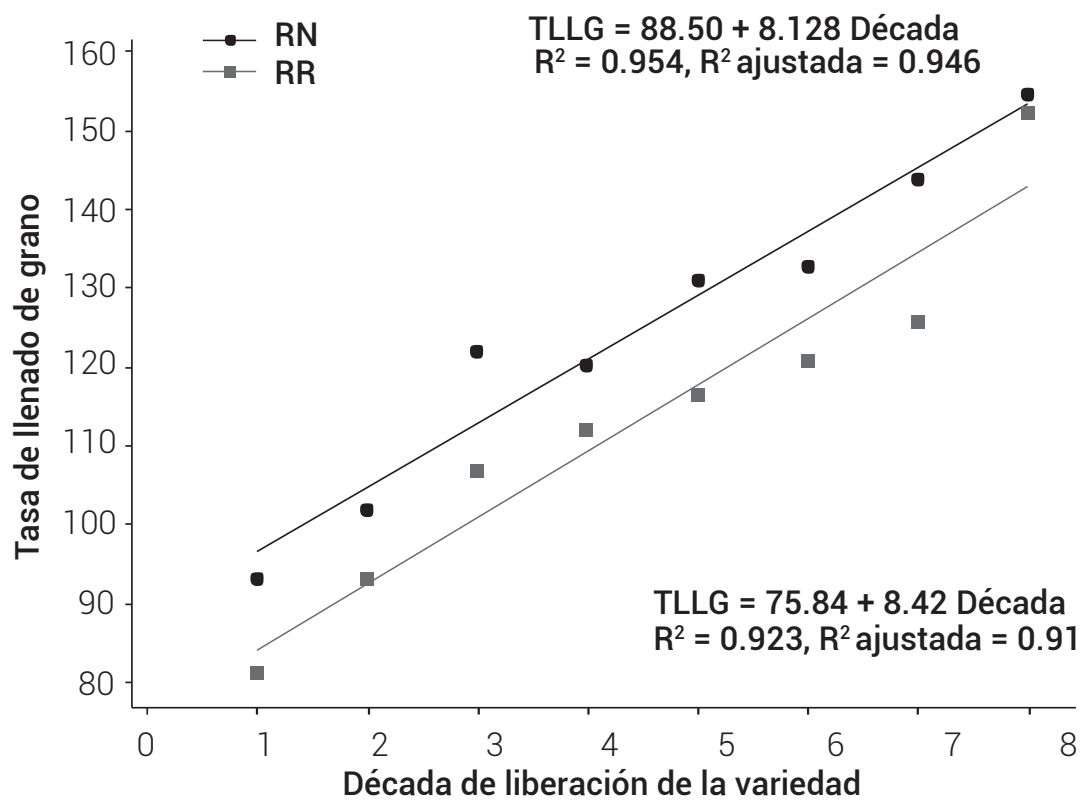

Figura 7. Relación entre la década de liberación de las variedades y tasa de llenado de grano ( $T L L G$ en $\mathrm{kg} \mathrm{ha}^{-1} \mathrm{~d}^{-1}$ ) bajo riego normal (RN) y restringido (RR) como promedio de los cuatro experimentos. Celaya, Guanajuato y Montecillo, Edo. de México, ciclo otoño-invierno de 2014 - 2015. 


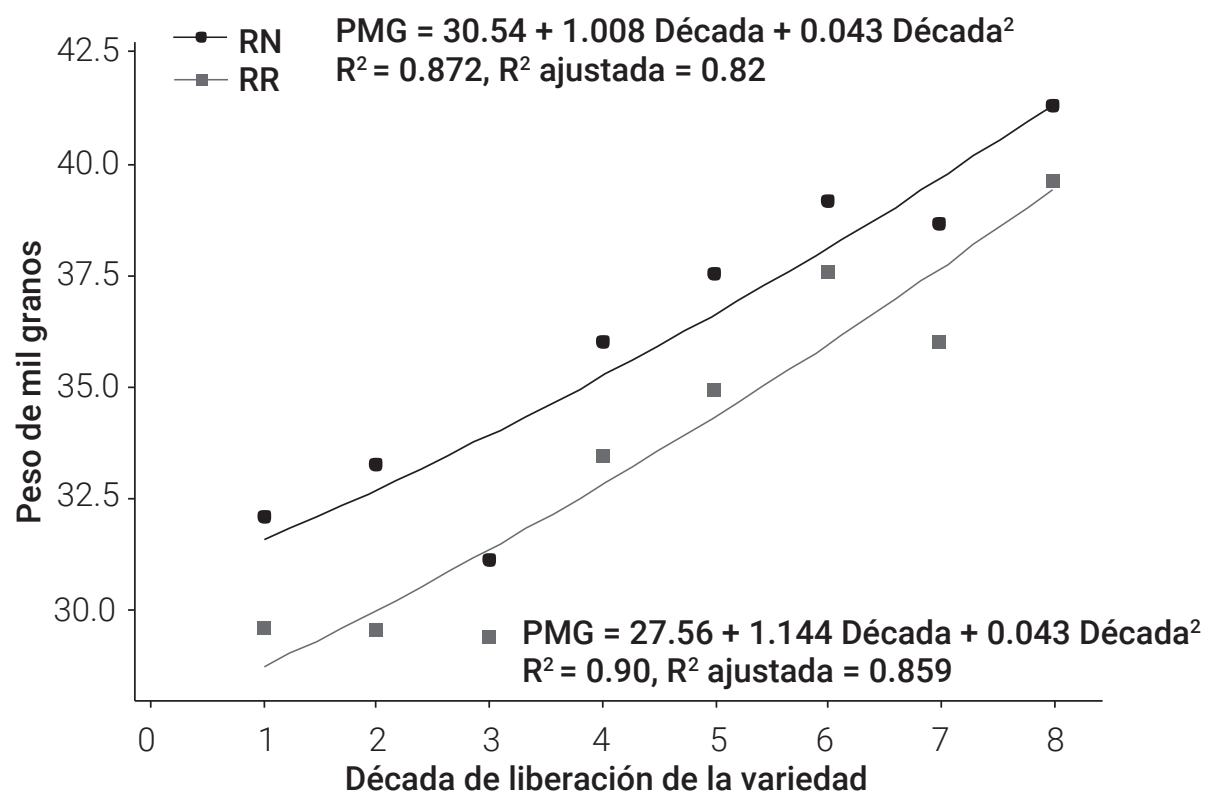

Figura 8. Relación entre la década de liberación de las variedades y peso de mil granos (PMG en g) bajo riego normal (RN) y restringido (RR) como promedio de los cuatro experimentos. Celaya, Guanajuato y Montecillo, Edo. de México, ciclo otoño-invierno de 2014 - 2015.

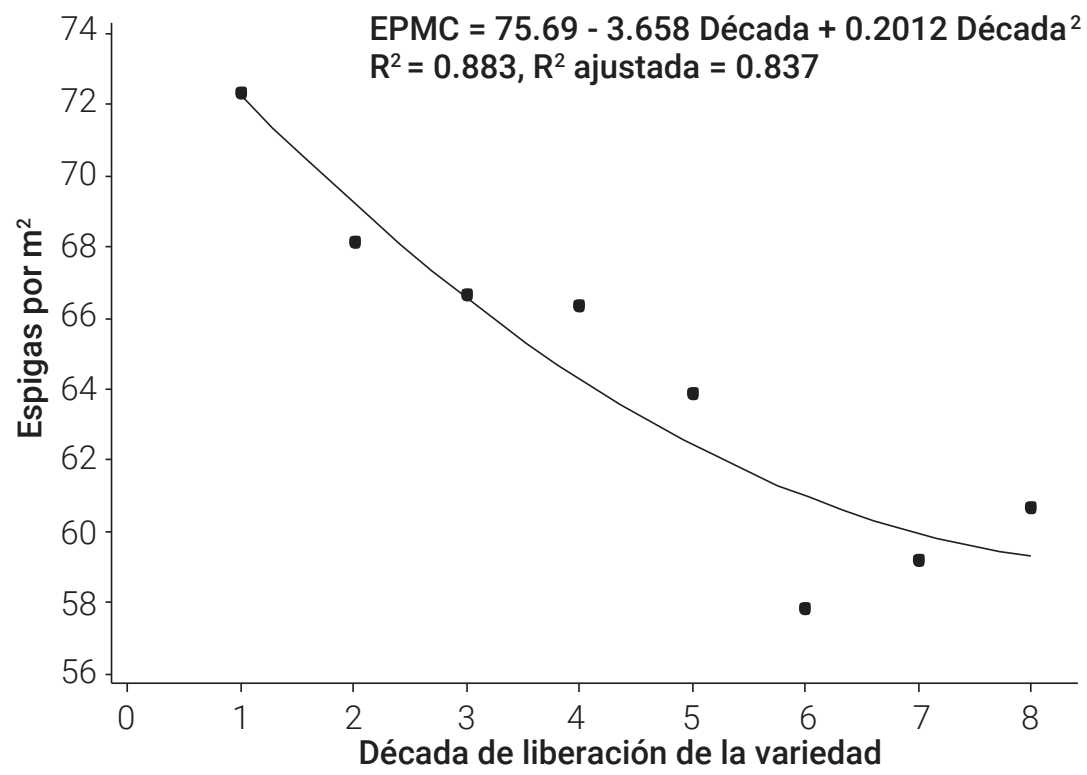

Figura 9. Relación entre la década de liberación de las variedades y espigas por $\mathrm{m}^{2}$ (EPMC) bajo riego normal (RN) como promedio de los cuatro experimentos. Celaya, Guanajuato y Montecillo, Edo. de México, ciclo otoño-invierno de 2014 2015.

normal y restringido para biomasa aérea, así como aumentar el número de espigas por $\mathrm{m}^{2}$ en los genotipos sobresalientes. Con el fin de seguir manteniendo el progreso genético del rendimiento de trigo, Fischer y Edmeades (2010) propusieron la mejora en el uso eficiente de la radiación que es una función de la tasa fotosintética y la distribución de la luz en el cultivo, porque ésta es la manera de aumentar la acumulación de biomasa que está ligada al progreso genético actual. Por otro lado, Lopes et al. (2012b) sugieren que la tasa de progreso incluso podría incrementarse en ambientes con estrés, si los rasgos de adaptación a la sequía y al calor son reunidos en un mismo genotipo. 


\section{CONCLUSIONES}

La ganancia del rendimiento de grano promedio fue 1.21 $\%$ anual $\left(41.77 \mathrm{~kg} \mathrm{ha}^{-1}\right)(\mathrm{P}<0.001)$ que en 67 años y a partir de las variedades liberadas en el año de 1948 representó un aumento acumulado de $2.8 \mathrm{t} \mathrm{ha}^{-1}$ logrado al seleccionar trigos con mayor peso de mil granos, mayor número de granos por $\mathrm{m}^{2} \mathrm{y}$ mayor biomasa aérea.

La tasa anual de progreso bajo riego restringido (1.35 \%) fue mayor que la obtenida bajo riego normal (1.10\%), lo que determina que las variedades modernas tienen mayor capacidad que las variedades antiguas de responder favorablemente al déficit hídrico.

Los rasgos más afectados por restringir el riego fueron rendimiento de grano, biomasa aérea y número de granos por metro cuadrado, con reducciones de $20.3,16.8$, y 14.5 $\%$, respectivamente.

\section{AGRADECIMIENTOS}

Al Consejo Nacional de Ciencia y Tecnología por la beca otorgada para realizar los estudios de Maestría en Ciencias. Al Fondo Sectorial SAGARPA-CONACYT, a través del proyecto No. 146788 por el financiamiento de la presente investigación.

\section{BIBLIOGRAFÍA}

Akram M. (2011) Growth and yield components of wheat under water stress of different growth stages. Bangladesh Journal of Agricultural Research 36:455-468.

Álvaro F., J. Isidro, D. Villegas, L. F. García del Moral and C. Royo (2008) Old and modern durum wheat varieties from Italy and Spain differ in main spike components. Field Crops Research 106:86-93.

Blum A. (2011) Plant Breeding for Water-Limited Environments. Springer. NY, USA. $267 \mathrm{p}$.

CANIMOLT, Cámara Nacional de la Industria Molinera de Trigo (2014) Indicadores del Trigo en México. http://www.canimolt.org/del-campoal-molino (Julio 2015)

CONAGUA, Comisión Nacional del Agua (2013) Atlas del Agua en México 2013. SEMARNAT. México, D.F. 144 p.

CONAGUA, Comisión Nacional del Agua (2015) Acuerdo por el que se actualiza la disponibilidad media anual de agua subterránea de los 653 acuíferos de los Estados Unidos Mexicanos, mismos que forman parte de las regiones hidrológico-administrativas que se indican. Diario Oficial. Segunda Sección. Poder Ejecutivo. Secretaría de Medio Ambiente y Recursos Naturales. www.dof.gob.mx/nota_detalle.php?codigo $=5389380 \&$ fec ha=20/04/2015 (Abril 2015)

Farooq M., M. Hussain and K. H. M. Siddique (2014) Drought stress in wheat during flowering and grain-filling periods. Critical Reviews in Plant Sciences 33:331-349.

Fischer R. A. and G. 0. Edmeades (2010) Breeding and cereal yield progress. Crop Science 50:S 85-S 98

Huerta-Espino J., E. H. Villaseñor-Mir, E. Espitia-Rangel, E. Solís-Moya and M. van Ginkel (2011) The history of wheat breeding in Mexico. In The World Wheat Book 2: A History of Wheat Breeding. Volume 2. A. William, J. Bonjean, M. van Ginkel (eds.). Lavoisier Publishing. Paris, France. pp:275-307.

IPCC, Intergovernmental Panel on Climate Change (2013) Annex I: Atlas of global and regional climate projections In: Climate Change
2013: The physical science basis. G. J. van Oldenborgh, M. CoIlins, J. Arblaster, J. H. Christensen, J. Marotzke, S. B. Power, M. Rummukainen and T. Zhou (eds.). Contribution of Working Group I to the Fifth Assessment Report of the Intergovernmental Panel of Climate Change. Stocker, T. F., D. Qin, G-K. Plattner, M. Tignor, S. K. Allen, J. Boschung, A. Nauels, Y. Xia, V. Bex and P. M. Midgley (eds.). Cambridge University Press. Cambridge, United Kingdom and New York, NY, USA. pp:1311-1393.

Isidro J., F. Álvaro, C. Royo, D. Villegas, D. J. Miralles and L. F. García del Moral (2011) Changes in duration of developmental phases of durum wheat caused by breeding in Spain and Italy during the 20th century and its impact in yield. Annals of Botany 107:1355-1366.

Ivanova A. and N. Tsenov (2011) Winter wheat productivity under favorable and drought environments. I. An overall effect. Bulgarian Journal of Agricultural Science 17:777-782.

Kaur V. and R. K. Behl (2010) Grain yield in wheat as affected by short periods of high temperature, drought and their interaction during pre- and post-anthesis stages. Cereal Research Communications 38:514-520

Lopes M. S. and M. P. Reynolds (2011) Drought adaptive traits and wide adaptation in elite lines derived from resynthesized hexaploid wheat. Crop Sience 51:1617-1626.

Lopes M. S., M. P. Reynolds, M. R. Jalal-Kamali, M. Moussa, Y. Feltaous, I. S. A Tahir, N. Barma, M. Vargas, Y. Mannes and M. Baum (2012b) The yield correlations of selectable physiological traits in a population of advanced spring wheat lines grown in warm and drought environments. Field Crops Research 128:129-136.

Lopes M. S., M. P. Reynolds, Y. Manes, R. P. Singh, J. Crossa and H. J. Braun (2012a) Genetic yield gains and changes in associated traits of CIMMYT spring bread wheat in a "Historic" set representing 30 years of breeding. Crop Science 52:1123-1131.

Madani A., A. S. Rad, A. Pazoki, G. Nourmohammadi and R. Zarghami (2010) Wheat (Triticum aestivum L.) grain filling and dry matter partitioning responses to source: sink modifications under postanthesis water and nitrogen deficiency. Acta Scientiarum Agronomy 32:145-151

Nalley L., A. P. Barkley and A. M. Featherstone (2010) The genetic and economic impact of the CIMMIYT wheat breeding program on local producers in the Yaqui Valley, Sonora, México. Agricultural Economics 41:453-462

Orta A. H., T. Erdem, S. Sehirali, Y. Erdem, I. Baser and O. Yorgancilar (2002) Water-yield relation and water use efficiency of Winter wheat in Western Turkey. Cereal Research Communications 30:367-374.

Prasad P. V. V., S. R. Pisipati, I. Momcilovic and Z. Ristic (2011) Independent and combined effects of high temperature and drought stress during grain filling on plant yield and chloroplast EF-Tu expression in spring wheat. Journal of Agronomy and Crop Science 197:430-441.

Reynolds M. P., Y. Manes y G. Rebetzke (2012) Aplicación de la fisiología en el fitomejoramiento para estrés por calor y sequía. In: Fitomejoramiento Fisiológico I: Enfoques Interdisciplinarios para Mejorar la Adaptación del Cultivo. M. P. Reynolds, A. Pask, D. Mullan. And P. Chávez (eds.). CIMMYT. México, D. F. pp:18-32.

Sánchez-García M., C. Royo, N. Aparicio, J. A. Martín-Sánchez and F. Álvaro (2013) Genetic improvement of bread wheat yield and associated traits in Spain during the 20th century. Journal of Agricultural Science 151:105-118.

Sayre K. D., R. P. Singh, J. Huerta-Espino and S. Rajaram (1998) Genetic prog ress in reducing losses to leaf rust in CIMMYT-Derived Mexican spring wheat cultivars. Crop Science 38:654-659.

SMN, Servicio Meteorológico Nacional (2015) Normales por estación http://smn.cna.gob.mx/index.php?option=com_content\&view =article\&id=176: chihuahua\&catid=14:normales-por-estacion. (Octubre 2015).

Shamsi K. and S. Kobraee (2011) Bread wheat production under drought stress conditions. Annals of Biological Research 2:352-358.

Singh R. P., J. Huerta-Espino and H. M. William (2005) Genetics and breeding for durable resistance to leaf and stripe rust in wheat. Turkish Journal of Agriculture and Forestry 29:121-127.

Solís-Moya E., J. Huerta-Espino, H. E. Villaseñor-Mir, P. Pérez-Herrera, A. Ramírez-Ramírez, L. Ledesma-Ramírez y M. L. de la Cruz-González (2013) Luminaria F2012, nueva variedad de trigo harinero para riego restringido en El Bajío. Folleto Técnico No. 21. INIFAP. Celaya, 
Gto., México. 32 p.

Suleiman A. A., J. F. Nganya and M. A. Ashraf (2014) Effect of cultivar and sowing date on growth and yield of wheat (Triticum aestivum L.) in Khartown, Sudan. Journal of Forest Products \& Industries 3:198-203.

Villaseñor M. H. E., J. Huerta E., P. Pérez H., M. F. Rodríguez G., E. Martínez C., R. Hortelano S. y E. Espitia R. (2009) La investigación de trigo en el Campo Experimental Valle de México: historia y aportaciones. In: Reseña Histórica, 66 Años de Investigación al Servicio de
México, 1943-2009. Campo Experimental Valle de México "El Horno". Publicación Especial No. 1. INIFAP. SAGARPA. México, D. F. pp:19-31.

Zadoks J. C., T. T. Chang and C. F. Konzak (1974) A decimal code for the growth stages of cereals. Weed Research 14:415-421.

Zhang J., Q. Mu and J. Huang (2016) Assessing the remotely sensed drought severity index for agricultural drought monitoring and impact analysis in North China. Ecological Indicators 63:296309 